\title{
Continuous Primary Care Is Central to Comprehensive Cancer Care: Are We Ready to Meet Growing Needs?
}

\author{
Shawna V. Hudson, PhD
}

Primary care engagement is essential to meet the need for high-quality, comprehensive, long-term cancer care. Primary care currently serves an integral role as the point of care for preventive and surveillance cancer screenings. As cancer prevalence rises, primary care will increasingly serve a growing need for care continuity as patients transition between screening, active treatment, and continued follow-up care. (J Am Board Fam Med 2013;26:623-625.)

\section{Continuous Primary Care is Central to Comprehensive Cancer Care: Are We Ready to Meet Growing Needs?}

Continuity of care and access to care are central issues for all patients. They can be critical for patients dealing with cancer. In this issue of the fournal of the American Board of Family Medicine, articles by Maly et $\mathrm{al}^{1}$ and Roetzheim et $\mathrm{al}^{2}$ address the centrality of primary care engagement in screening for cancer and continued follow-up after cancer treatment.

Roetzheim and colleagues ${ }^{2}$ studied cancer screening for melanoma in a Medicare population who reported visits with both a dermatologist and primary care physician (PCP). They found that patients who visited either both a dermatologist and PCP or a PCP alone were more likely to be diagnosed at an earlier stage and with thinner melanomas, thereby improving melanoma mortality

From Rutgers, the State University of New Jersey, Biomedical and Health Sciences; the Department of Family Medicine and Community Health, Robert Wood Johnson Medical School; and the Rutgers Cancer Institute of New Jersey, Somerset, NJ.

Funding: none.

Conflict of interest: none declared.

Corresponding author: Shawna Hudson, $\mathrm{PhD}$, Department of Family Medicine and Community Health, Rutgers Robert Wood Johnson Medical School, 1 World's Fair Dr., Suite 1500, Somerset, NJ 08873 (E-mail: hudsonsh@ rutgers.edu).

See Related Articles on Pages 628 and 637. among these patients. These findings point to continuity of care in primary care as being a central factor that influences whether patients are seen often enough to provide adequate opportunities to screen. Maly et $\mathrm{al}^{1}$ similarly studied PCP-directed management of follow-up preventive screening after a breast cancer diagnosis. They also report that primary care is integral to the receipt of screening for breast cancer surveillance and preventive colorectal and cervical cancer screening, particularly among a low-income population. Indeed, they find that women who saw only a PCP and not a cancer specialist for their follow-up care had the highest odds of receiving each clinical service. Together, these studies highlight the necessity and centrality of primary care in the management of cancer through providing both preventive and surveillance cancer screening.

These 2 studies bring to the fore a new and growing challenge for primary care: How can PCPs manage the increasing numbers of cancer survivors in their patient panels and meet their complex ongoing needs for screening and surveillance? There are approximately 13.7 million cancer survivors in the United States, and by January 1, 2022, that number is projected to increase to nearly 18 million. ${ }^{3}$ Most current cancer survivors were diagnosed more than 5 years ago (64\%), with approximately $15 \%$ diagnosed more than 20 years ago. ${ }^{3}$ Current data suggest that these trends will continue. Breast and melanoma cancers are among the most prevalent and have some of the highest rates of cure. ${ }^{3}$ More than $60 \%$ of breast cancers and 3 of 4 melanoma cancers are diagnosed and treated at 
localized stages. ${ }^{3}$ As far as these patients and their doctors know, they have been "cured," which means they are highly unlikely to continue making regular trips to see their specialists and cancer treatment teams for follow-up.

We know from previous research that the further patients get from their active or curative cancer treatment, the more likely they are to be cared for exclusively in a primary care setting. A population-based study using Surveillance, Epidemiology, and End Results data linked to Medicare claims found that after 5 years of survival only one third of long-term cancer survivors continued to seek care from physicians whose specialties are related to their original cancer. ${ }^{4}$ Approximately $46 \%$ of longterm breast cancer survivors were followed by their oncologists at year 5, yet their rates fell to $11 \%$ in their 12th year of survival. They also found that patients with localized disease were significantly more likely to be followed by their primary care providers. In contrast, only $20 \%$ of survivors report receiving the majority of their health care from a cancer specialist. ${ }^{5}$ Results from Maly et $\mathrm{al}^{1}$ in this issue of the fournal underscore this reality and suggest that for low-income populations it may not be feasible to continue follow-up care in an oncology setting, regardless of patient preference.

It is therefore imperative for primary care to continue to remain engaged and play an active role in the coordinated care of cancer survivors. This is a population at increased risk for additional comorbidities and complications resulting from their prior cancer treatments as well as accelerated aging if their comorbid health risks are not appropriately managed. ${ }^{6}$ Moreover, primary care is well positioned for intervention with those cancer survivors who do not engage in protective health behaviors, including those who continue to smoke (15\%), do not participate in adequate physical activity (32\%), and are obese $(28 \%))^{5}$ Cancer survivors are also at increased risk for both recurrence as well as new second primary cancers. Therefore, it is paramount that they continue to receive preventive and surveillance screening. However, we know that many are not receiving recommended preventive care, not only for cancer screening but also for influenza and pneumococcal vaccinations. ${ }^{5}$ In our study of cancer survivors in community practice settings, we found that although rates of preventive cancer screening among cancer survivors were higher than those among patients without a history of cancer, they were lower than optimal given cancer survivors' increased risk of cancer recurrence and/or second primary cancer. ${ }^{7}$ Furthermore, their selfreports of screening were higher than the actual testing documented in their medical records, suggesting that there may be some confusion about what constitutes cancer screening. For these reasons, it is imperative for primary care to engage in actively monitoring cancer survivors through the use of health maintenance and prevention visits.

Although there is increased need for monitoring cancer survivor follow-up in primary care, there is some concern about the quality of monitoring provided. There are a number of articles that point to limited PCP information and expertise to deal with cancer treatment-related late effects or unknown long-term effects of treatment. Maly et $\mathrm{al}^{1}$ describe this as a potential barrier. However, what much of the existing literature fails to take into account is that in many health care markets throughout the country where there is low specialty penetration or where access to specialists are constricted by limited resources, primary care is already dealing with these issues without the benefit of an extensive safety net. The articles by both Maly et al and Roetzheim et $\mathrm{al}^{2}$ address this gap in the literature. Roetzheim et al raise this issue when they suggest that even though dermatologists may provide more accurate skin examinations in contrast with PCPs, their impact may be more limited because they have fewer contacts with patients and therefore fewer opportunities to diagnose melanoma at earlier stages. Roetzheim et al present data consistent with this argument: more than half of the patients diagnosed with melanoma in their study had contact with their PCP - not a dermatologist-in the 2 years leading to their diagnosis. Seizing opportunities for diagnosing cancer at earlier stages has important implications for cancer survival, which in turn shapes the needs of the long-term cancer survivor population.

The studies by Maly et $\mathrm{al}^{1}$ and Roetzheim et $\mathrm{al}^{2}$ remind us of the centrality of engaging primary care in providing effective and timely screening for prevention and as part of continued follow-up after cancer treatment. Both raise important questions about what next steps should be taken to further enhance uptake of preventive screening for cancer and other conditions in primary care. These studies focus on the ends of the screening continuum from prevention before a cancer diagnosis to surveillance 
afterward during survivorship care and underscore the need for primary care to play a continuous role as part of comprehensive cancer care. Baby boomers and cancer survivors will have an increasing need for these services in the next decade. Thus, we must make certain that we in primary care are ready to meet their growing needs.

\section{References}

1. Maly RC, Liu Y, Diamant AL, Thind A. The impact of primary care physicians on follow-up care of underserved breast cancer survivors. J Am Board Fam Med 2013;26:628-36.

2. Roetzheim RG, Lee J-H, Ferrante JM, et al. The influence of dermatologist and primary care physician visits on melanoma outcomes among medicare beneficiaries. J Am Board Fam Med 2013;26:637-47.
3. Siegel R, Desantis C, Virgo K, et al. Cancer treatment and survivorship statistics, 2012. CA Cancer $\mathrm{J}$ Clin 2012;62:220-41.

4. Pollack LA, Adamache W, Ryerson AB, Eheman CR, Richardson LC. Care of long-term cancer survivors: physicians seen by Medicare enrollees surviving longer than 5 years. Cancer 2009;115:5284-95.

5. Underwood JM, Townsend JS, Stewart SL, et al. Surveillance of demographic characteristics and health behaviors among adult cancer survivors-Behavioral Risk Factor Surveillance System, United States, 2009. MMWR Surveill Summ 2012;61:1-23.

6. Ganz PA. The 'three Ps' of cancer survivorship care. BMC Med 2011;9:14.

7. Hudson SV, Hahn KA, Ohman-Strickland P, Cunningham RS, Miller SM, Crabtree BF. Breast, colorectal and prostate cancer screening for cancer survivors and non-cancer patients in community practices. J Gen Intern Med 2009;24(Suppl 2):S487-90. 